Research Paper:

\title{
The Relationship Between COVID-19-induced Death and Chronic Diseases
}

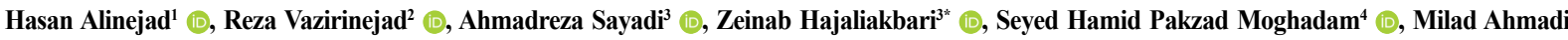
Gohari $\odot$, Mahdi Alinejad ${ }^{6}$ (1), Zinat Sadat Dehghan Menshadi ${ }^{7}$ (1)

1. Department of Government Management, Rafsanjan University of Medical Science, Rafsanjan, Iran.

2. Department of Epidemiology, School of Medicine, Rafsanjan University of Medical Science, Rafsanjan, Iran.

3. Department of Nursing, Faculty of Nursing and Midwifery, Rafsanjan University of Medical Sciences, Rafsanjan, Iran.

4. Department of Anesthesiology, School of Medicine, Rafsanjan University of Medical Sciences, Rafsanjan, Iran.

5. Department of Biostatistics, Modeling in Health Research Center, Institute for Futures Studies in Health, Kerman University of Medical Sciences, Kerman, Iran 6. Department of Accounting, Yazd Branch, Azad Islamic University, Yazd, Iran.

7. Department of Emergency Medicine, Faculty of Emergency Medicine, Rafsanjan University of Medical Science, Rafsanjan, Iran.

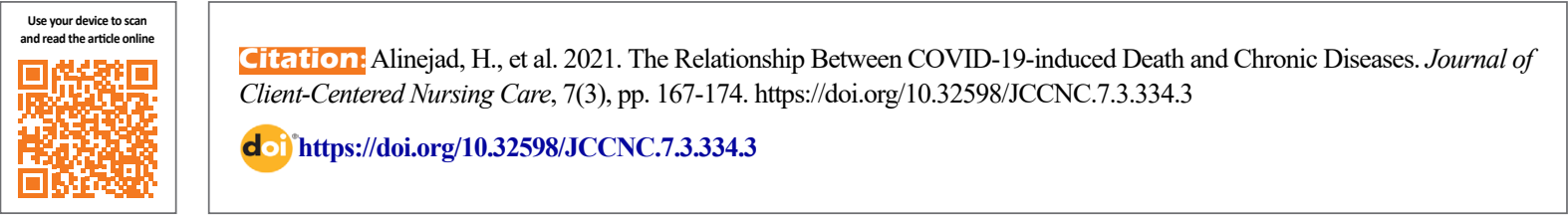

\section{(i) (\$)}

Article info:

Received: 12 Jun 2021

Accepted: 06 Jul 2021

Published: 01 Aug 2021
Keywords:

Chronic diseases, Coronavirus Disease 2019 (COVID-19), In hospital mortality, Mortality determinants

\section{A B S T RA C T}

Background: Morbidity and mortality due to Coronavirus Disease 2019 (COVID-19) are mainly related to age and comorbidity diseases (hypertension, diabetes, cardiovascular disease, malignancies, etc.). These conditions are associated with poorer clinical outcomes and sometimes lead to long-term hospitalization. The current study aimed to investigate the relationship between COVID-19-induced mortality and various chronic diseases in patients admitted to Ali Ibn Abitaleb Hospital in Rafsanjan City, Iran, in 2020.

Methods: In this retrospective, descriptive, and cross-sectional study, patients with COVID-19 referring to Ali Ibn Abitaleb Hospital in Rafsanjan City, Iran, from March 2020 to September 2020 were assessed. The required data were collected using patients' records and telephone calls by a researcher-made checklist and analyzed by Independent Samples t-test, Chi-squared test, Fisher's Exact test, Kaplan-Meier plots, and multivariate regression analysis in SPSS v. 20.

Results: This study assessed 238 hospitalized patients with COVID-19. The risk of death was significantly higher in patients aged over 75 years; they were 5.5 times more prone to expire, compared to the youngest age group $(\mathrm{P}<0.001)$. Chronic diseases, such as hypertension, heart disease, lung disease, and various cancers were more prevalent in patients who expired, compared to those who survived $(\mathrm{P} \leq 0.05)$. Of the patients who died, $73.8 \%$ were transferred to the Intensive Care Unit (ICU), while only $7.5 \%$ of surviving patients were transferred to the ICU $(\mathrm{P}<0.001)$. Longer hospitalization was associated with an increased risk of death among patients with underlying diseases and hypertension $(\mathrm{P}<0.05)$.

Conclusion: This study identified the role of chronic diseases and other important indicators in the survival of patients with COVID-19 who were admitted to a hospital in Rafsanjan. It is recommended that nurses and healthcare staff consider these findings in the care of patients with COVID-19.

* Corresponding Author:

Zeinab Hajaliakbari, MSc.

Address: Department of Nursing, Faculty of Nursing and Midwifery, Rafsanjan University of Medical Sciences, Rafsanjan, Iran Tel: +98 (914) 2187767

E-mail:zhrooz@yahoo.com 


\section{Highlights}

- The mean age of patients with COVID-19 who expired and survived was 72.21 and 56.61 years, respectively.

- The prevalence of chronic diseases was higher in patients who died, compared to those who survived.

- Chronic diseases, such as hypertension, heart disease, lung disease, and various cancers were more prevalent in patients who died than the survivors.

- No significant relationship was found between gender and COVD-19-related mortality.

- The most common signs and symptoms of COVID-19 were shortness of breath, fever, body aches, headache, cough, decreased sense of smell and taste, vomiting, diarrhea, and loss of consciousness, respectively.

- The survival of patients aged over 75 years decreased by $40 \%$, compared to patients under 49 years of age over a ten-day period.

\section{Plain Language Summary}

The morbidity and mortality of COVID-19 are related to many factors. This study assessed the relationship between COVID-19- mortality and various chronic diseases, such as hypertension, cardiovascular disease, cancer, and diabetes in patients admitted to Ali Ibn Abitaleb Hospital in Rafsanjan City, Iran, in 2020. The study findings suggested that the elderly and those with chronic conditions are more frequently hospitalized, admit to the ICU, and were expired due to COVID-19. Furthermore, longer hospital stays were associated with higher mortality.

\section{Introduction}

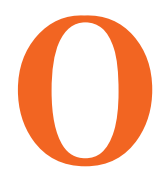

n March 11, 2019, the World Health Organization announced the outbreak of a new coronavirus, called Coronavirus Disease 2019 (COVID-19) as a pandemic (Lai et al. 2020). COVD1-19 is usually associated with respiratory disease and its most common clinical features are fever, fatigue, and dry cough. Some patients may experience pain, nasal congestion, runny nose, sore throat, or diarrhea. In more severe cases, the infection can cause pneumonia, acute respiratory syndrome, and sometimes death (Geleta et al. 2020). However, $80 \%$ of patients with COVID-19 may only present mild to moderate symptoms. The mortality rate is variable according to test methods, access to the healthcare system, and other unknown factors. Reports generally suggested a $2 \%-7 \%$ mortality; it may be up to $20 \%$ in the elderly (Verity et al. 2020).

Morbidity and mortality due to COVID-19 are related to age and comorbidity diseases (hypertension, diabetes, cardiovascular disease, malignancies, etc.). Besides, these diseases lead to poorer clinical outcomes and sometimes longer hospitalizations (Rothan \& Byrareddy 2020). A meta-analysis of patients with COVID-19 and chronic conditions indicated that those with hypertension, diabetes, respiratory disease, cardiovascular disease, and other chronic diseases are more prone to COVID-19 infection (Yang et al. 2020). Evidence revealed that chronic illness increases the risk of severe COVID-19 as well as the need for hospitalization, Intensive Care Unit (ICU) admission, and intubation (Almalki et al. 2020). Some studies explored the association between COVID-19 and chronic diseases. Regarding the importance of this subject and given the limited research on this issue in Iran, it was essential to perform this study. Therefore, this study aimed at assessing the relationship between COVID-19-related mortality and chronic diseases in Rafsanjan City, Iran, in 2020.

\section{Materials and Methods}

This was a retrospective, descriptive, and cross-sectional study. The research population was all patients with COVID-19 referring to Ali Ibn Abitaleb Hospital in Rafsanjan City, Iran. A total of 238 patients with COVID-19 were admitted to Ali Ibn Abitaleb Hospital in Rafsanjan from March to September 2020. The information was obtained by reviewing the records and making telephone calls with the patients. Telephone contacts were made with patients to complete the information 
not recorded in the files. The obtained data included age, gender, the presence of chronic disease, the length of hospital stay, signs and symptoms and their duration, death due to the disease, and hospitalization in the ICU, i.e., recorded in a researcher-made checklist. This checklist was approved by the related experts. The necessary data were collected in 45 days and analyzed by Independent Samples t-test, Chi-squared test, Fisher's Exact test, Kaplan-Meier plots, and multivariate regression analysis using SPSS v. 20.

\section{Results}

This study assessed the records of 238 hospitalized patients with COVID-19. The mean age of those who died, compared to those who survived was 72.21 vs 56.61 $(\mathrm{P}<0.001)$. The incidence of chronic diseases, such as hypertension, heart disease, lung disease, and various cancers was higher in patients who expired, compared to those who survived $(\mathrm{P} \leq 0.05)$. Diabetes was more prevalent in patients who died than those who survived; however, this difference was borderline concerning significance $(\mathrm{P}=0.07)$. Among the patients who died, $73.8 \%$ were transferred to the ICU, while only $7.5 \%$ of those who survived were transferred to the ICU $(\mathrm{P}<0.001)$. No significant relationship was found between gender and mortality due to COVID-19 (Table 1). The most common signs and symptoms of COVID-19 were shortness of breath (73.5\%), fever (59.7\%), body aches (58.4\%), headache (34\%), cough (32.4\%), decreased sense of smell and taste $(24.4 \%)$, vomiting $(22.3 \%)$, diarrhea (20.3\%), and loss of consciousness (12.2\%), in sequence (Table 2). Kaplan-Meier plot for the prognostic factors of COVID-19, i.e., statistically significant or borderline is presented in Figure 1. This graph illustrates that the odds of death among individuals with underlying diseases and hypertension increases over time $(\mathrm{P}<0.05)$.

The odds of survival among patients with heart and lung diseases and cancer decreased over time; however, this reduction was statistically borderline $(\mathrm{P}=\sim 0.08)$. The graph demonstrates that the patients who were transferred to the ICU presented a $50 \%$ lower odds of survival after about 10 days, compared to the other patients $(\mathrm{P}<0.001)$. This graph also suggests that after about 10 days of hospitalization, the odds of the survival of patients, aged over 75 years, compared to those under 49 years of age, decreased by approximately $40 \%(\mathrm{P}<0.001)$. Multivariate Cox regression analysis was applied to examine the risk ratio (Table 3). Multivariate models containing statistically significant variables were statistically significant $\left(\mathrm{x}^{2}=25.92, \mathrm{df}=9, \mathrm{P}<0.01\right)$ In the presence of other vari-

Table 1. Distribution of the expired and surviving patients by important indicators

\begin{tabular}{|c|c|c|c|c|}
\hline \multirow{2}{*}{ Important Indicators } & \multicolumn{3}{|c|}{ No.(\%) } & \multirow{2}{*}{$\mathbf{P}$} \\
\hline & Total & Expired & Survived & \\
\hline Male & $120(50.4)$ & $20(52.6)$ & $100(50)$ & 0.77 \\
\hline Chronic Disease & 142(59.66) & $33(86.8)$ & $109(54.5)$ & $<0.001$ \\
\hline Hypertension & $68(28.6)$ & 18(47.4) & $50(25.0)$ & $<0.05$ \\
\hline Diabetes & $65(27.3)$ & $15(39.5)$ & $50(25.0)$ & 0.07 \\
\hline Heart Disease & $43(18.1)$ & 12(31.6) & $31(15.5)$ & 0.02 \\
\hline Thyroid Disease & $12(5.0)$ & $1(2.6)$ & $11(4.5)$ & 0.46 \\
\hline Cancers & $9(3.8)$ & $4(10.5)$ & $5(2.5)$ & 0.04 \\
\hline Lung Disease & $44(18.5)$ & $13(34.2)$ & $31(15.5)$ & $<0.01$ \\
\hline Alzheimer disease & $8(3.4)$ & $2(5.3)$ & $6(3)$ & 0.62 \\
\hline Normal & $79(33.2)$ & $18(47.4)$ & $61(30.5)$ & 0.04 \\
\hline $\mathrm{ICU}$ & $43(18.1)$ & $28(73.8)$ & $15(7.5)$ & $<0.001$ \\
\hline Total & $238(100)$ & $38(15.97)$ & $200(84.03)$ & - \\
\hline
\end{tabular}


Table 2. Distribution of deceased and surviving patients according to the signs and symptoms

\begin{tabular}{|c|c|c|c|c|}
\hline \multirow{2}{*}{ Signs and Symptoms } & \multicolumn{3}{|c|}{ No. (\%) } & \multirow{2}{*}{$\mathbf{P}$} \\
\hline & Total & Expired & Survived & \\
\hline Dyspnea & $175(73.5)$ & $28(73.8)$ & $147(73.8)$ & $0.98^{*}$ \\
\hline Cough & $77(32.4)$ & $7(18.4)$ & $70(35)$ & 0.04 \\
\hline Diarrhea & $48(20.2)$ & $2(5.3)$ & $46(23)$ & $0.01^{* *}$ \\
\hline Vomiting & $53(22.3)$ & $7(18.4)$ & $46(23)$ & 0.53 \\
\hline Vertigo & 19(8) & $0(0)$ & 19(9.5) & $0.05^{* *}$ \\
\hline Fever & $142(59.7)$ & $13(34.2)$ & $129(64.5)$ & $<0.001$ \\
\hline Anosmia & $58(24.4)$ & $0(0)$ & $58(29)$ & $<0.001^{* *}$ \\
\hline Lack of taste & $58(24.4)$ & $0(0)$ & $58(29)$ & $<0.001^{* *}$ \\
\hline Body ache & $139(58.4)$ & $7(18.4)$ & $132(66)$ & $<0.001$ \\
\hline Headache & $81(34)$ & $2(5.3)$ & $79(39.5)$ & $<0.001^{* *}$ \\
\hline Decreased level of consciousness & $29(12.2)$ & $17(44.7)$ & $12(6)$ & $<0.001$ \\
\hline Total & $238(100)$ & $38(15.97)$ & 200(84.03) & - \\
\hline
\end{tabular}

Table 3. The hazard ratio for fatal outcomes

\begin{tabular}{|c|c|c|c|}
\hline \multicolumn{2}{|c|}{ General Characteristics } & \multirow{2}{*}{$\begin{array}{c}\text { Hazard Ratio }(95 \% \mathrm{Cl}) \\
\text { Ref }\end{array}$} & \multirow[t]{2}{*}{$\mathbf{P}$} \\
\hline & $\leq 49$ & & \\
\hline \multirow[t]{2}{*}{ Age (y) } & $50-74$ & $1.88(0.55,6.42)$ & 0.3 \\
\hline & $\geq 75$ & $5.50(1.60,18.31)$ & $<0.01$ \\
\hline \multirow{11}{*}{ Gender } & Female & Ref & \\
\hline & Male & $1.17(0.58,2.38)$ & 0.66 \\
\hline & Chronic disease & $1.92(0.57,6.43)$ & 0.29 \\
\hline & Hypertension & $1.55(0.70,3.43)$ & 0.28 \\
\hline & Diabetes & $1.15(0.54,2.49)$ & 0.71 \\
\hline & Heart disease & $0.87(0.40,1.88)$ & 0.71 \\
\hline & Thyroid disease & $1.72(0.21,13.86)$ & 0.61 \\
\hline & Cancers & $1.83(0.58,5.79)$ & 0.30 \\
\hline & Lung disease & $0.96(0.43,2.12)$ & 0.91 \\
\hline & Alzheimer's disease & $0.72(0.15,3.51)$ & 0.69 \\
\hline & Normal & $1.51(0.75,3.05)$ & 0.25 \\
\hline
\end{tabular}



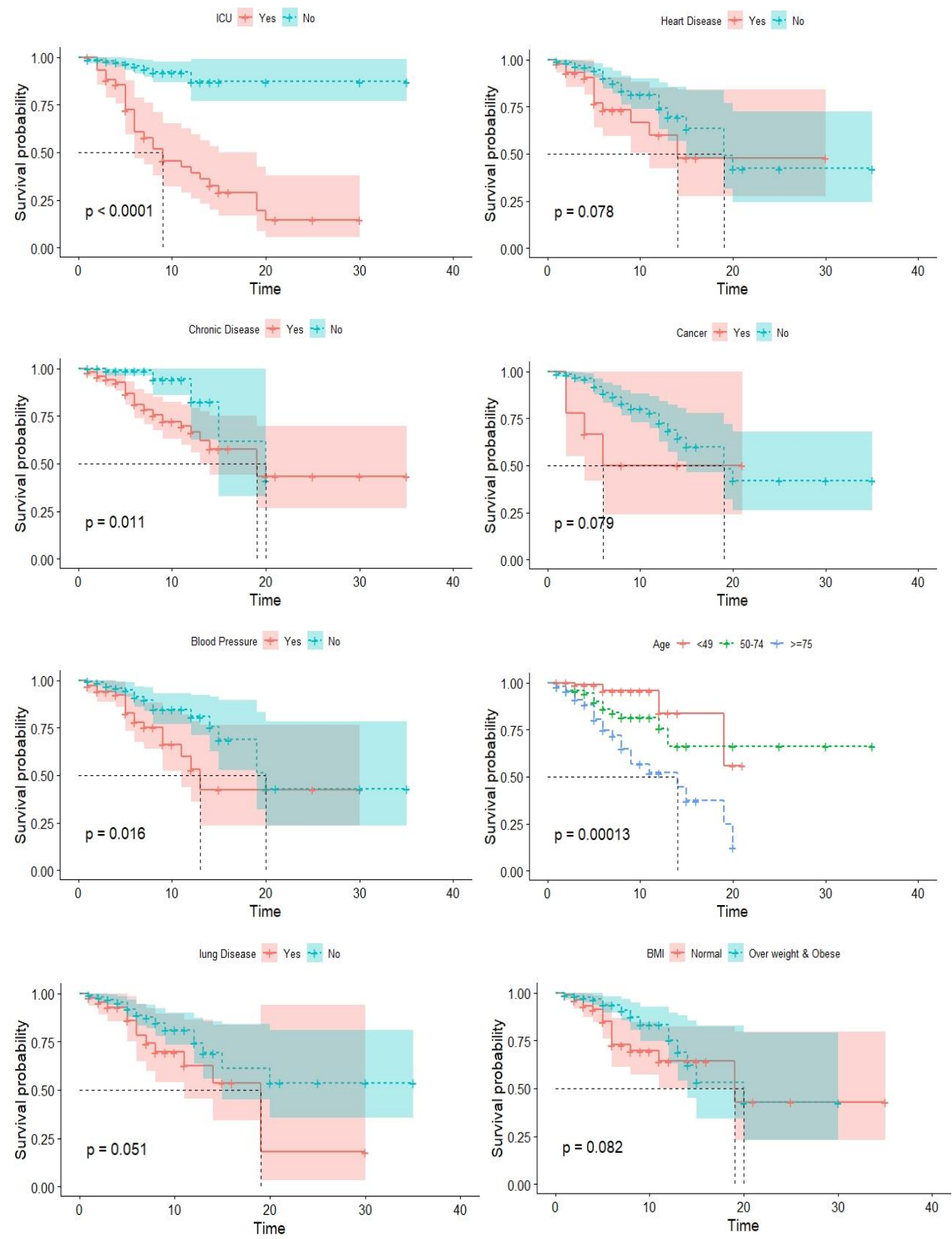

Figure 1. Kaplan-Meier survival plots for different prognostic factors

Client- Centered Nursing Care

ables, only the effect of age was significant. The risk of death was significantly higher in individuals aged over 75 years $(\mathrm{P}<0.01)$. They were 5.5 times more prone to die than the youngest age group. Although having factors, such as hypertension, diabetes, cancers, thyroid disease, and male gender increased the risk of death, these values were not statistically significant.

\section{Discussion}

This retrospective descriptive, cross-sectional, and single-center study assessed the clinical characteristics and chronic underlying diseases of 238 hospitalized patients with COVID-19 and their relationship with the patients' mortality. According to the obtained results, the mean age of patients who died from COVID-19, compared to those who survived was 72.21 and 56.61 years, respectively $(\mathrm{P}<0.001)$. Other studies revealed that aging in patients with COVID-19 is associated with higher mortality (Zhou et al. 2020). A study in Japan indicated that the mortality rate among COVID-19 patients, aged 70-79 years and over 80 years was equal to $6.8 \%$ and $14.8 \%$, respectively; however, COVID19-induced mortality in all other age groups was mea- 
sured as $2.6 \%$ (Shibata et al. 2020). Another study revealed that patients aged over 65 years have constituted $45 \%$ of hospitalizations, $53 \%$ of the ICU admissions, and $80 \%$ of all deaths due to COVID-19 (Wang et al. 2020b). Decreased immune function and low levels of immune response in the elderly might lead to higher mortality in these individuals (Wu et al. 2020).

The prevalence of chronic diseases, such as hypertension, heart disease, lung disease, and various cancers in our study was higher in patients who died, compared to those who survived $(\mathrm{P}<0.05)$. Furthermore, the prevalence of diabetes was higher in the group of patients who died, compared to the survivors; however, this value was statistically borderline $(\mathrm{P}=0.07)$. Other studies revealed that chronic diseases, such as cardiovascular disease (Yang et al. 2020; Soares et al. 2020), cancer (Soares et al. 2020; He et al. 2020), hypertension, and lung disease (Zhou et al. 2020) have led to more mortality in patients with COVID-19. A study published in March 2020 found that $19.5 \%$ of those with COVID-19 who died in Italy had active cancer (Onder et al. 2020). A UK study of 800 patients with COVID-19 and cancer found that half of the patients had mild COVID-19 and the mortality was significantly related to the patients' age and their comorbid conditions, such as hypertension and cardiovascular disease (Lee et al. 2020). In a meta-analysis of 6 studies with 1527 patients, hypertension and cardiovascular diseases were present in $17.1 \%$ and $16.4 \%$ of the population, respectively ( $\mathrm{Li}$ et al. 2020). Zhou et al. stated that $45 \%$ of those with hypertension who died of COVID-19 have accounted about $28 \%$ of all COVID-19 deaths (Zhou et al. 2020).

A study in Italy found that patients with diabetes were up to $50 \%$ more prone to die from COVID-19 than the others (Remuzzi \& Remuzzi 2020). Other studies signified hypertension and diabetes as the most common chronic diseases associated with COVID-19 and have a high mortality rate (Salam 2016). High blood glucose adversely affects clinical outcomes and reduces the effects of antiviral drugs (Sardu et al. 2020; Marfella et al. 2020). In the current study, among the patients who died, $73.8 \%$ were transferred to the ICU, while only $7.5 \%$ of those who survived were transferred to the ICU $(\mathrm{P}<0.001)$; patients who were transferred to the ICU had a $50 \%$ lower chance of survival after about 10 days, compared to those who were not transferred to that ward $(\mathrm{P}<0.001)$.

A study by Hasse et al. in Denmark found that the mortality rate in the ICU was $37 \%$. This rate increased with a longer stay in the ICU; however, half of the patients who were hospitalized in the ICU for 4 weeks or more survived and were discharged from the hospital (Haase et al. 2021). Some other studies indicated higher mortality than survival in COVID-19 patients admitted to the ICU (Docherty et al. 2020). In a study on patients with COVID-19 who were admitted to the ICU in China, the mortality rate equaled 39\% (Wang et al. 2020c). Grasselli et al., in a study in Lombardy, Italy revealed that $87.3 \%$ of the subjects with COVID-19 were transferred to the ICU and $48.7 \%$ of them died (Grasselli et al. 2020).

A study on 138 hospitalized COVID-19 patients in Wuhan, China reported that the average age of those who were transferred to the ICU was 65 years, compared with 51 years in those who received no intensive care. It was also found that $72.2 \%$ of the patients admitted to the ICU had comorbidities, while $37.3 \%$ of those who were not admitted to the ICU had comorbidities (Wang et al. 2020a). In our study, there was no significant relationship between gender and mortality due to COVID-19. A study by Bhopal et al. in London, England, found that the gender ratio of male to female mortality was generally 1.4 , which varied by age. This ratio was equal to 0.81 for the age of $0-9$ years, 1.9 for the age of 40-49 years, 2.3 for the age of 50-59 years, 2.6 for the age of 60-69 years, and 1.65 for the age range of over 80 years (Bhopal \& Bhopal 2020). In this study, the most common signs and symptoms of COVID-19 were as follows: shortness of breath, fever, body aches, headache, cough, decreased sense of smell and taste, vomiting, diarrhea, loss of consciousness, and vertigo, respectively. A study in Taiwan reported mild fever, shortness of breath, and mild hypoxemia as common symptoms of the disease (Cheng et al. 2020).

A study in Turkey investigated two groups of patients suspected to having COVID-19. The most common symptoms in the group with COVID-19 were cough $(56.6 \%)$, weakness $(56.6 \%)$, taste disturbance $(35.7 \%)$, myalgia (34.3\%), and fever (33.6\%); in individuals without COVID-19 symptoms, the symptoms included cough (63\%), weakness $(45.5 \%)$, dyspnea $(29.9 \%)$, and headache $(24.7 \%)$. In this study, taste and smell disorders as well as diarrhea were significantly higher in the positive COVID-19 group (Çalıca Utku et al. 2020). In a study in France, 7 symptoms were present in more than half of the patients, as follows: fatigue $(93 \%)$, cough $(80 \%)$, fever $(77 \%)$, headache $(73 \%)$, myalgia $(59 \%)$, arthralgia (54\%), and anosmia (53\%) (Klopfenstein et al. 2020).

This was a single-center study and several patients did not cooperate in the data collection process. Additionally, the study population differed in terms of economic and health status as well as access to medical services; therefore, the obtained data may not reflect the whole population. 


\section{Conclusion}

The current study results suggested that COVID-19 was significantly associated with older ages, chronic diseases, longer hospitalization, ICU admission, and mortality. Mortality increased as the length of stay was extended in the ICU. Individuals with chronic illnesses, such as hypertension, diabetes, heart disease, and cancer are also more prone to die over time. It is recommended that nurses and other members of the health care team consider these findings in the care of patients with COVID-19.

\section{Ethical Considerations}

\section{Compliance with ethical guidelines}

The study protocol was approved by the Ethics Committee of Rafsanjani University of Medical Sciences (Code: IR.RUMS.REC.1398.164). Consent was obtained for using the files of the patients.

\section{Funding}

This study was financially supported by the Center for the Study of Social Factors Affecting the Health affiliated with the Medical School of Rafsanjan University of Medical Sciences.

\section{Authors' contributions}

Conceptualization and supervision: Hasan Alinejad, and Zeinab Hajaliakbari; Data collection: Zeinab Hajaliakbari and Ahmadreza Sayadi; Data analysis: Milad Ahmadi; Writing - original draft, and writing - review \& editing: Zeinab Hajaliakbari; Final approval: All authors.

\section{Conflict of interest}

The authors declared no conflicts of interest.

\section{Acknowledgments}

We want to thank all the Rafsanjan University of Medical Sciences colleagues and patients who participated in this project.

\section{References}

Almalki, Z. S., et al. 2020. Clinical characteristics and outcomes among COVID-19 hospitalized patients with chronic conditions: A retrospective single-center study. Journal of Multidisciplinary Healthcare, 13, pp. 1089-97. [DOI:10.2147/JMDH.S273918]
Bhopal, S. S. \& Bhopal, R., 2020. Sex differential in COVID-19 mortality varies markedly by age. The Lancet, 396(10250), pp 532-3. [DOI:10.1016/S0140-6736(20)31748-7]

Çalıca Utku, A., et al. 2020. Main symptoms in patients presenting in the COVID-19 period. Scottish Medical Journal, 65(4), pp. 127-32. [DOI:10.1177/0036933020949253]

Capuano, A., et al. 2020. NSAIDs in patients with viral infections, including Covid-19: Victims or perpetrators? Pharmacological Research, 157, p. 104849. [DOI:10.1016/j.phrs.2020.104849]

Cheng, S. C., et al. 2020. First case of Coronavirus Disease 2019 (COVID-19) pneumonia in Taiwan. Journal of the Formosan Medical Association, 119(3), pp. 747-51. [DOI:10.1016/j. jfma.2020.02.007]

Docherty, A. B., et al. 2020. Features of 20133 UK patients in hospital with COVID-19 using the ISARIC WHO Clinical Characterisation Protocol: Prospective observational cohort study. BMJ, 22, p. 369. [DOI:10.1136/bmj.m1985]

Foo, Y. Z., et al. 2017. The effects of sex hormones on immune function: A meta-analysis. Biological Reviews, 92(1), pp. 551-71. [DOI:10.1111/brv.12243]

Geleta, T. A., et al. 2020. COVID-19 Pandemic preparedness and response of chronic disease patients in public health facilities. International Journal of General Medicine, 13, p. 1011. [DOI:10.2147/IJGM.S279705]

Grasselli, G., et al. 2020. Risk factors associated with mortality among patients with COVID-19 in intensive care units in Lombardy, Italy. JAMA Internal Medicine, 180(10), pp. 1345-55. [DOI:10.1001/jamainternmed.2020.3539]

Gupta, S., et al. 2020. Factors associated with death in critically ill patients with Coronavirus disease 2019 in the US. JAMA Internal Medicine, 180(11), pp. 1436-47. [DOI:10.1001/jamainternmed.2020.3596]

Haase, N., et al. 2021. Characteristics, interventions, and longer term outcomes of COVID-19 ICU patients in Denmark-A nationwide, observational study. Acta Anaesthesiologica Scandinavica, 65(1), pp. 68-75. [DOI:10.1111/aas.13701]

He, F., et al. 2020. Clinical features and risk factors for ICU admission in COVID-19 patients with cardiovascular diseases. $\mathrm{Ag}$ ing and Disease, 11(4), pp. 763-9. [DOI:10.14336/AD.2020.0622]

Klein, S. L., Jedlicka, A. \& Pekosz, A., 2010. The Xs and Y of immune responses to viral vaccines. The Lancet Infectious Diseases, 10(5), pp. 338-49. [DOI:10.1016/S1473-3099(10)70049-9]

Klopfenstein, T., et al. 2020. New loss of smell and taste: Uncommon symptoms in COVID-19 patients in Nord Franche-Comte cluster, France. International Journal of Infectious Diseases, 100, pp. 117-22. [DOI:10.1016/j.ijid.2020.08.012]

Lai, C. C., et al. 2020. Asymptomatic carrier state, acute respiratory disease, and pneumonia due to severe acute respiratory syndrome Coronavirus 2 (SARS-CoV-2): Facts and myths. Journal of Microbiology, Immunology and Infection, 53(3), pp. 40412. [DOI:10.1016/j.jmii.2020.02.012]

Lee, L. Y., et al. 2020. COVID-19 mortality in patients with cancer on chemotherapy or other anticancer treatments: A prospective cohort study. The Lancet, 395(10241), pp. 1919-26. [DOI:10.1016/S0140-6736(20)31173-9] 
Li, B., et al. 2020. Prevalence and impact of cardiovascular metabolic diseases on COVID-19 in China. Clinical Research in Cardiology, 109(5), pp. 531-8. [DOI:10.1007/s00392-020-01626-9]

Marfella, R., et al. 2020. Negative impact of hyperglycaemia on tocilizumab therapy in COVID -19 patients. Diabetes $\mathcal{E}$ Metabolism, 46(5), pp. 403-5. [DOI:10.1016/j.diabet.2020.05.005]

Onder, G., Rezza, G. \& Brusaferro, S., 2020. Case-fatality rate and characteristics of patients dying in relation to COVID-19 in Italy. JAMA, 323(18), pp. 1775-6. [DOI:10.1001/jama.2020.4683]

Remuzzi, A. \& Remuzzi, G. 2020. COVID-19 and Italy: What next? The Lancet, 395(10231), pp. 1225-8. [DOI:10.1016/S01406736(20)30627-9]

Rothan, H. A. \& Byrareddy, S. N., 2020. The epidemiology and pathogenesis of Coronavirus disease (COVID-19) outbreak. Journal of Autoimmunity, 109, p. 102433. [DOI:10.1016/j. jaut.2020.102433]

Roved, J., Westerdahl, H. \& Hasselquist, D., 2017. Sex differences in immune responses: Hormonal effects, antagonistic selection, and evolutionary consequences. Hormones and Behavior, 88, pp. 95-105. [DOI:10.1016/j.yhbeh.2016.11.017]

Salam, R., 2016. Expanding the definition of noncommunicable disease. Journal of Social Health and Diabetes, 4(2), pp. 67-70. [DOI:10.4103/2321-0656.187990]

Sardu, C., et al. 2020. Impact of diabetes mellitus on clinical outcomes in patients affected by COVID-19. Cardiovascular Diabetology, 19, p. 76. [DOI:10.1186/s12933-020-01047-y]

Shibata, S., et al. 2020. Hypertension and related diseases in the era of COVID-19: A report from the Japanese Society of Hypertension Task Force on COVID-19. Hypertension Research, 43, pp. 1028-46. [DOI:10.1038/s41440-020-0515-0]

Soares, R. D. C. M., Mattos, L. R. \& Raposo, L. M., 2020. Risk factors for hospitalization and mortality due to COVID-19 in Espírito Santo State, Brazil. The American Journal of Tropical Medicine and Hygiene, 103(3), pp. 1184-90. [DOI:10.4269/ ajtmh.20-0483]

Suleyman, G., et al. 2020. Clinical characteristics and morbidity associated with Coronavirus disease 2019 in a series of patients in metropolitan Detroit. JAMA Network Open, 3(6), p. e2012270. [DOI:10.1001/jamanetworkopen.2020.12270]

Verity, R., et al. 2020. Estimates of the severity of Coronavirus disease 2019: A model-based analysis. The Lancet Infectious Diseases, 20(6), pp. 669-77. [DOI:10.1016/S1473-3099(20)30243-7]

Wang, D., et al. 2020a. Clinical characteristics of 138 hospitalized patients with 2019 novel Coronavirus-infected pneumonia in Wuhan, China. JAMA, 323(11), pp. 1061-9. [DOI:10.1001/ jama.2020.1585]

Wang, W., Tang, J. \& Wei, F., 2020b. Updated understanding of the outbreak of 2019 novel Coronavirus (2019-nCoV) in Wuhan, China. Journal of Medical Virology, 92(4), pp. 441-7. [DOI:10.1002/jmv.25689]

Wang, Y., et al. 2020. Clinical course and outcomes of 344 intensive care patients with COVID-19. American Journal of Respiratory and Critical Care Medicine, 201(11), pp. 1430-34. [DOI:10.1164/rccm.202003-0736LE]

Wu, C., et al. 2020. Risk factors associated with acute respiratory distress syndrome and death in patients with Coronavirus disease 2019 pneumonia in Wuhan, China. JAMA Internal Medicine, 180(7), pp. 934-43. [DOI:10.1001/jamainternmed.2020.0994]
Yang, J., et al. 2020. Prevalence of comorbidities and its effects in patients infected with SARS-CoV-2: A systematic review and meta-analysis. International Journal of Infectious Diseases, 94, pp. 91-5. [DOI:10.1016/j.ijid.2020.03.017]

Zhou, F., et al. Clinical course and risk factors for mortality of adult inpatients with COVID-19 in Wuhan, China: A retrospective cohort study. The Lancet, 395(10229), pp. 1054-62. [DOI:10.1016/S0140-6736(20)30566-3] 